\title{
Geometrical Formulation of 3-D Space-Time Finite Integration Method
}

\author{
$\operatorname{AUTHOR}(\mathrm{S})$ :
}

Kawahara, Jun; Mifune, Takeshi; Matsuo, Tetsuji

\section{CITATION:}

Kawahara, Jun ...[et al]. Geometrical Formulation of 3-D Space-Time Finite Integration Method. IEEE Transactions on Magnetics 2013, 49(5): 1693-1696

\section{ISSUE DATE:}

2013-05

URL:

http://hdl.handle.net/2433/174348

\section{RIGHT:}

(c) 2013 IEEE. Personal use of this material is permitted. Permission from IEEE must be obtained for all other uses, in any current or future media, including reprinting/republishing this material for advertising or promotional purposes, creating new collective works, for resale or redistribution to servers or lists, or reuse of any copyrighted component of this work in other works.; この論文は出版社版でありません。引用の際には出版社版をご確認ご利用ください。;This is not the published version. Please cite only the published version. 


\title{
Geometrical Formulation of 3D Space-Time Finite Integration Method
}

\author{
Jun Kawahara, Takeshi Mifune, and Tetsuji Matsuo, Member, IEEE \\ Graduate School of Engineering, Kyoto University, Kyoto 615-8510, Japan
}

\begin{abstract}
A geometrical formulation of a space-time finite integration (FI) method is studied for application to electromagnetic wave propagation calculations. Based on the Hodge duality and Lorentzian metric, a modified relation is derived between the incidence matrices of space-time primal and dual grids. A systematic method to construct the Maxwell grid equations on the space-time primal and dual grids is developed. The geometrical formulation is implemented on a simple space-time grid, which is proven equivalent to an explicit time-marching scheme of the space-time FI method.
\end{abstract}

Index Terms-Finite integration method, graph theory, Hodge duality, space-time grid.

\section{INTRODUCTION}

$\mathbf{T}$ HE finite integration (FI) method [1]-[5] has been studied to accomplish time-domain electromagnetic field computations using unstructured spatial grids. The FI method derives grid-based Maxwell equations using incidence matrices based on the dual computational-grid geometry. Graph theory enables a systematic construction of the spatial dual grid from the primal grid geometry. However, the geometry description is restricted to the spatial domain. Accordingly, similar to the FDTD method [6], the FI method uses a uniform time-step, which is restricted by the Courant-Friedrichs-Lewy condition [7] based on the smallest spatial grid size.

Previous work [8] introduced a space-time FI method that achieves non-uniform time-steps naturally on the three- dimensional (3D) space-time grid with 2D space. The Hodge dual grid was proposed in [9] to construct the $4 \mathrm{D}$ space-time grid for electromagnetic field computation. An application of space-time FI method to a photonic band computation was reported in [12]. However, it is not always a simple task to construct the Maxwell grid equations on these dual spacetime grids. To realize a systematic derivation of Maxwell grid equations, a graph-theory-based formulation for the space-time dual grids is required. This paper discusses a geometrical formulation of the 3D space-time FI method that is based on the Hodge duality and the Lorentzian metric but is not a straightforward extension of the conventional spatial FI formulation.

\section{Finite Integration Method on a Space-Time GRID}

\section{A. Electromagnetics in Space-Time}

The Maxwell equations are described in the differential form as

$$
\mathrm{d} F=0, \quad \mathrm{~d} G=J .
$$

Manuscript received October 31, 2012; revised December 27, 2012 (date on which paper was submitted for review). Corresponding author: T. Matsuo (email: tmatsuo@kuee.kyoto-u.ac.jp).

Digital Object Identifier inserted by IEEE
In the coordinate system $\left(x^{0}, x^{1}, x^{2}, x^{3}\right)=(t, x, y, z), F, G$ and $J$ are written as

$$
\begin{aligned}
F & =-\Sigma_{i=1}^{3} E_{i} \mathrm{~d} x^{0} \mathrm{~d} x^{i}+\Sigma_{j=1}^{3} B_{j} \mathrm{~d} x^{k} \mathrm{~d} x^{l}, \\
G & =\Sigma_{i=1}^{3} H_{i} \mathrm{~d} x^{0} \mathrm{~d} x^{i}+\Sigma_{j=1}^{3} D_{j} \mathrm{~d} x^{k} \mathrm{~d} x^{l}, \\
J & =c \rho \mathrm{d} x^{1} \mathrm{~d} x^{2} \mathrm{~d} x^{3}-\Sigma_{j=1}^{3} c J_{j} \mathrm{~d} x^{0} \mathrm{~d} x^{k} \mathrm{~d} x^{l}
\end{aligned}
$$

where $c$ is the speed of light, $\rho$ is the electric charge density and $(j, k, l)$ is a cyclic permutation of $(1,2,3)$. The integral form of (1) is given as

$$
\oint_{\partial \Omega_{\mathrm{p}}} F=0, \quad \oint_{\partial \Omega_{\mathrm{d}}} G=\int_{\Omega_{\mathrm{d}}} J
$$

where $\Omega_{\mathrm{p}}$ and $\Omega_{\mathrm{d}}$ are hypersurfaces in space-time.

For simplicity, assuming the uniformity along the $z$ direction, this paper discusses the FI formulation for the electromagnetic field $\left(B_{z}, E_{x}, E_{y}\right)$ in the $(w, x, y)-3 \mathrm{D}$ free space-time [8], where $w=$ ct. Accordingly, $F$ and $G$ are written as

$$
\begin{aligned}
& F=B_{z} \mathrm{~d} x \mathrm{~d} y+\mathcal{E}_{y} \mathrm{~d} y \mathrm{~d} w-\mathcal{E}_{x} \mathrm{~d} w \mathrm{~d} x, \\
& G=\mathcal{H}_{z} \mathrm{~d} w-D_{y} \mathrm{~d} x+D_{x} \mathrm{~d} y
\end{aligned}
$$

where $\left(\mathcal{E}_{x}, \mathcal{E}_{y}\right)=\left(E_{x} / c, E_{y} / c\right), \mathcal{H}_{z}=H_{z} / c$. Defining 3D vectors $\boldsymbol{F}$ and $\boldsymbol{G}$ as

$$
\boldsymbol{F}=\left(B_{z}, \mathcal{E}_{y},-\mathcal{E}_{x}\right), \quad \boldsymbol{G}=\left(\mathcal{H}_{z},-D_{y}, D_{x}\right)
$$

the integral form is written without source term as

$$
\oint_{S} \boldsymbol{F} \cdot \boldsymbol{n} \mathrm{d} S=0, \quad \oint_{C} \boldsymbol{G} \cdot \boldsymbol{t} \mathrm{d} s=0
$$

where $\boldsymbol{n}$ is the normal vector at each point on the closed surface $S$ and $t$ the tangent vector at each point on the closed curve $C$. The Euclidean metric is used for the dot product operation.

The space-time FI method uses discretized variables as

$$
f=\int_{p} \boldsymbol{F} \cdot \boldsymbol{n} \mathrm{d} S, \quad g=\int_{\tilde{s}} \boldsymbol{G} \cdot \boldsymbol{t} \mathrm{d} s
$$


where $p$ is a face of a primal grid and $\tilde{s}$ is an edge of a dual grid. To express the constitutive equation between $f$ and $g$ simply, $\boldsymbol{n}$ and $\boldsymbol{t}$ are given as [8]

$$
\boldsymbol{n}=\left(n_{w}, n_{x}, n_{y}\right), \quad \boldsymbol{t}=\left(n_{w},-n_{x},-n_{y}\right) .
$$

Fig. 1 illustrates the geometrical relation between $\boldsymbol{n}$ and $\boldsymbol{t}$, where $\tilde{s}$ is orthogonal to $p$ in the Lorentzian 3D space-time. The relation between $\boldsymbol{F} \cdot \boldsymbol{n}$ and $\boldsymbol{G} \cdot \boldsymbol{t}$ is given as

$$
\boldsymbol{F} \cdot \boldsymbol{n}=Z \boldsymbol{G} \cdot \boldsymbol{t}
$$

where $Z$ is the impedance of the medium. Thus, $f$ is related to $g$ as

$$
\begin{aligned}
& f=z g \\
& z=Z \frac{\Delta S}{\Delta l}
\end{aligned}
$$

where $\Delta S$ is the area of $p$ and $\Delta l$ is the length of $\tilde{s}$.

Ref. [9] extended the dual-grid construction above to the 4D space-time, called the Hodge dual grid. It is based on the Hodge duality with the Lorentzian metric between $F$ and $G$, where the metric is modified in materials depending on the speed of light.
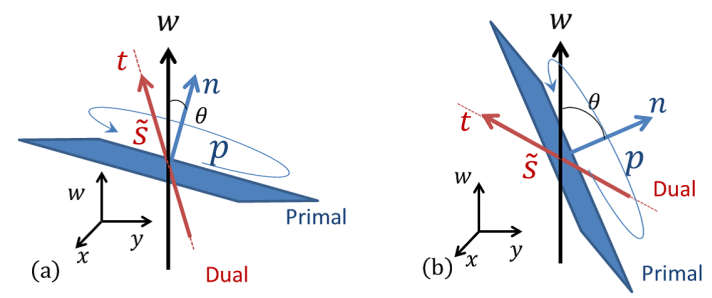

Fig. 1: Relation of primal face and dual edge in space-time grid when (a) $\boldsymbol{n} \cdot \boldsymbol{t}>0$ and (b) $\boldsymbol{n} \cdot \boldsymbol{t}<0$.

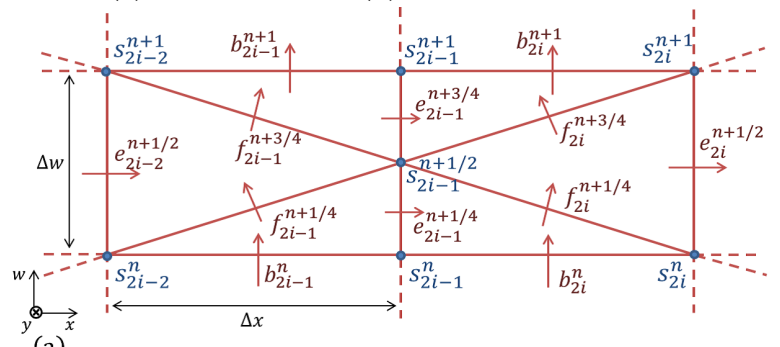

(a)

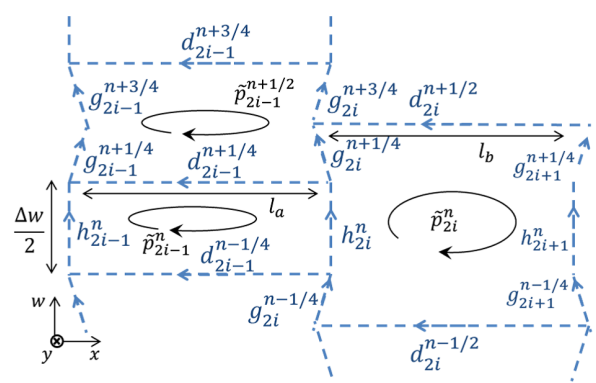

Fig. 2: Edges and faces on (a) the primal grid and (b) the dual grid.

\section{B. Explicit Time-Marching Scheme}

Refs. [8] and [9] have shown explicit time-marching schemes for 3D and 4D space-time FI analyses of electromagnetic wave propagation. This subsection presents an explicit time-marching scheme on a simple 2D space-time grid with 1D space to relate to the geometrical formulation described later.

Fig. 2 illustrates 2D space-time primal and dual grids that have temporal step sizes $\Delta w$ and $\Delta w / 2$ and spatial step size $\Delta x$ along the $x$ - and $y$ - directions.

Based on (5) and (7), the variables in Fig. 2 have the following meaning; $b$ : magnetic flux, $e$ : electromotive force, $f$ : the composition of $b$ and $e, h$ : magnetomotive force, $d$ : electric flux, and $g$ : the composition of $h$ and $d$. The arrow directions in Fig. 2 are based on (7) and (9) using the definition (5) and (8). Note that the arrow direction of $d$ is opposite to that of $e$. These do not correspond directly to the directions of $\boldsymbol{E}$ and $D$ in the Euclidean space.

The explicit time-marching scheme is given as follows. According to a numerical examination in [10], the scheme is stable when $\left(l_{a}-1\right)^{2}+(\Delta w)^{2} / 2<1$.

Variables $d_{2 i-1}^{n+1 / 4}$ and $e_{2 i-1}^{n+1 / 4}$ are given as

$$
\begin{aligned}
& d_{2 i-1}^{n+1 / 4}=d_{2 i-1}^{n-1 / 4}-\left(h_{2 i}^{n}-h_{2 i-1}^{n}\right) \\
& e_{2 i-1}^{n+1 / 4}=z_{e 1} d_{2 i-1}^{n+1 / 4}, \quad z_{e 1}=Z \frac{\Delta w \Delta x}{2 l_{a}} .
\end{aligned}
$$

On the primal grid, $f_{2 i-1}^{n+1 / 4}, f_{2 i}^{n+1 / 4}$ and consequently $g_{2 i-1}^{n+1 / 4}$, $g_{2 i}^{n+1 / 4}$ are given as

$$
\begin{aligned}
f_{2 i-1}^{n+1 / 4} & =-e_{2 i-1}^{n+1 / 4}+b_{2 i-1}^{n}, \\
f_{2 i}^{n+1 / 4} & =e_{2 i-1}^{n+1 / 4}+b_{2 i}^{n} \\
g_{k}^{n+1 / 4}=\frac{1}{z_{f}} f_{k}^{n+1 / 4}(k & =2 i-1,2 i), z_{f}=Z \frac{4 \Delta x^{2}}{\Delta w} .
\end{aligned}
$$

On the dual grid, $d_{2 i}$ and $e_{2 i}$ are updated using

$$
\begin{aligned}
d_{2 i}^{n+1 / 2}= & d_{2 i}^{n-1 / 2}+h_{2 i}^{n}-h_{2 i+1}^{n} \\
& +g_{2 i}^{n-1 / 4}-g_{2 i+1}^{n-1 / 4}+g_{2 i}^{n+1 / 4}-g_{2 i+1}^{n+1 / 4} \\
e_{2 i}^{n+1 / 2}= & z_{e 2} d_{2 i}^{n+1 / 2}, z_{e 2}=Z \frac{\Delta w \Delta x}{2-l_{a}+(\Delta w)^{2} / 4} .
\end{aligned}
$$

Similarly, $f_{2 i-1}^{n+3 / 4}, f_{2 i}^{n+3 / 4}$ and $g_{2 i-1}^{n+3 / 4}, g_{2 i}^{n+3 / 4}$ are given as

$$
\begin{aligned}
f_{2 i-1}^{n+3 / 4} & =f_{2 i-1}^{n+1 / 4}+e_{2 i-2}^{n+1 / 2}, \\
f_{2 i}^{n+3 / 4} & =f_{2 i}^{n+1 / 4}-e_{2 i}^{n+1 / 2} \\
g_{k}^{n+3 / 4} & =\frac{1}{z_{f}} f_{k}^{n+3 / 4}(k=2 i-1,2 i) .
\end{aligned}
$$

On the dual grid, $d_{2 i-1}^{n+3 / 4}$ and $e_{2 i-1}^{n+3 / 4}$ are obtained from

$$
\begin{aligned}
d_{2 i-1}^{n+3 / 4}= & d_{2 i-1}^{n+1 / 4}+g_{2 i-1}^{n+1 / 4}-g_{2 i}^{n+1 / 4} \\
& +g_{2 i-1}^{n+3 / 4}-g_{2 i}^{n+3 / 4} \\
e_{2 i-1}^{n+3 / 4}= & z_{e 1} d_{2 i-1}^{n+3 / 4} .
\end{aligned}
$$

Hence, $b_{2 i-1}^{n+1}, b_{2 i}^{n+1}$ and $h_{2 i-1}^{n+1}, h_{2 i}^{n+1}$ are given by

$$
\begin{aligned}
b_{2 i-1}^{n+1} & =f_{2 i-1}^{n+3 / 4}-e_{2 i-1}^{n+3 / 4}, \\
b_{2 i}^{n+1} & =f_{2 i}^{n+3 / 4}+e_{2 i-1}^{n+3 / 4} \\
h_{k}^{n}=\frac{1}{z_{b}} b_{k}^{n}(k & =2 i-1,2 i), \quad z_{b}=Z \frac{2 \Delta x^{2}}{\Delta w} .
\end{aligned}
$$




\section{Incidence Matrices on 3D Euclidean Space}

The FI method is generally formulated with the Maxwell grid equations using the incidence matrices from graph theory.

Let arrays $\{n\},\{s\},\{p\}$ and $\{v\}$ denote the sets of nodes, edges, faces, and volumes in the primal grid, respectively. These are related by incidence matrices $[G],[C]$ and $[D][1]$, [2], [11] as

$$
\partial\{s\}=[G]\{n\}, \partial\{p\}=[C]\{s\}, \partial\{v\}=[D]\{p\}
$$

where $\partial$ denotes restriction to the boundary. Similarly, the sets of nodes, edges, faces and volumes in the dual grid are related as

$$
\partial\{\tilde{s}\}=[\tilde{G}]\{\tilde{n}\}, \partial\{\tilde{p}\}=[\tilde{C}]\{\tilde{s}\}, \partial\{\tilde{v}\}=[\tilde{D}]\{\tilde{p}\} .
$$

In the Euclidean space, the dual grid is generally constructed so the incidence matrices satisfy

$$
[\tilde{C}]=[C]^{\mathrm{T}},[\tilde{D}]=-[G]^{\mathrm{T}},[\tilde{G}]=-[D]^{\mathrm{T}} .
$$

The relation above derives the Maxwell grid equations systematically from the primal grid geometry.

The space-time primal and dual grids based on (8) have a similar property to that described by (26), which gives the one-to-one correspondence between the faces $\{p\}$ on the primal grid and the edges $\{\tilde{s}\}$ on the dual grid. However, the directional relation between $\{p\}$ and $\{\tilde{s}\}$ determined by (8) differs from that given by (26). The following subsection derives the matrix relation for the space-time primal and dual grids based on (8).

\section{Incidence Matrices on 3D Space-Time}

A simple space-time primal grid illustrated in Fig. 3 is examined. Assuming spatial periodicity in the grid geometry, the edges $s_{i}$ and faces $p_{i}$ are periodically numbered for notational simplicity. Moreover, the edges and faces perpendicular to the $y$-axis is omitted. The direction of edges $s_{1}, s_{2}, s_{3}, s_{1}^{\prime}$, $s_{2}^{\prime}$ is along the $+y$-direction. The edge $\tilde{s}_{i}$ on the dual grid corresponds to the face $p_{i}$ on the primal grid, where their directions satisfy (8).
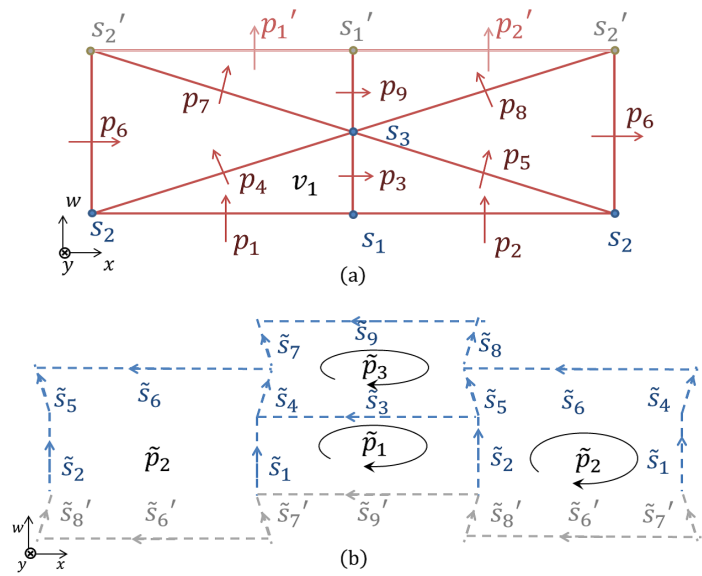

Fig. 3: Edges and faces on (a) the primal grid and (b) the dual grid.
The geometrical relation between edges and faces on the primal grid is represented by the following equations.

$$
\begin{aligned}
& \partial p_{1}=s_{1}-s_{2}, \partial p_{2}=-s_{1}+s_{2}, \partial p_{3}=s_{1}-s_{3}, \\
& \partial p_{4}=-s_{2}+s_{3}, \partial p_{5}=s_{2}-s_{3}, \partial p_{6}=s_{2}-s_{2}^{\prime}, \\
& \partial p_{7}=s_{3}-s_{2}^{\prime}, \partial p_{8}=-s_{3}+s_{2}^{\prime}, \partial p_{9}=s_{3}-s_{1}^{\prime} .
\end{aligned}
$$

To examine the relation between $[C]$ and $[\tilde{C}]$, a subset of $\{s\}$ and a subset of $\{p\}$ are defined as

$$
\{s\}_{\mathrm{sb}}=\left[\begin{array}{lll}
s_{1} & s_{2} & s_{3}
\end{array}\right]^{\mathrm{T}},\{p\}_{\mathrm{sb}}=\left[\begin{array}{llll}
p_{1} & p_{2} & \cdots & p_{9}
\end{array}\right]^{\mathrm{T}} .
$$

Omitting $s_{1}^{\prime}, s_{2}^{\prime}$, relation (27) is written $\{p\}_{\mathrm{sb}}=[C]_{\mathrm{sb}}\{s\}_{\mathrm{sb}}$ where $[C]_{\mathrm{sb}}$ is a submatrix of $[C]$ and given as

$$
[C]_{\mathrm{sb}}^{\mathrm{T}}=\left[\begin{array}{ccccccccc}
1 & -1 & 1 & 0 & 0 & 0 & 0 & 0 & 0 \\
-1 & 1 & 0 & -1 & 1 & 1 & 0 & 0 & 0 \\
0 & 0 & -1 & 1 & -1 & 0 & 1 & -1 & 1
\end{array}\right] .
$$

On the dual grid, the relation between edges and faces is found to be:

$$
\begin{aligned}
& \partial \tilde{p}_{1}=\tilde{s}_{1}-\tilde{s}_{2}-\tilde{s}_{3}+\tilde{s}_{9}^{\prime}, \\
& \partial \tilde{p}_{2}=-\tilde{s}_{1}+\tilde{s}_{2}-\tilde{s}_{4}+\tilde{s}_{5}-\tilde{s}_{6}+\tilde{s}_{6}^{\prime}-\tilde{s}_{7}^{\prime}+\tilde{s}_{8}^{\prime}, \\
& \partial \tilde{p}_{3}=\tilde{s}_{3}+\tilde{s}_{4}-\tilde{s}_{5}+\tilde{s}_{7}-\tilde{s}_{8}-\tilde{s}_{9} .
\end{aligned}
$$

Corresponding to $\{s\}_{\mathrm{sb}}$ and $\{p\}_{\mathrm{sb}}$, subsets of $\{\tilde{p}\}$ and $\{\tilde{s}\}$ are defined as

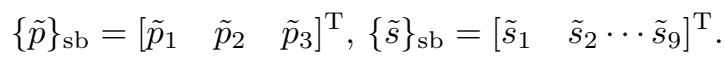

Omitting $\tilde{s}_{6}^{\prime}, \tilde{s}_{7}^{\prime}, \tilde{s}_{8}^{\prime}, \tilde{s}_{9}^{\prime}$, relation (30) is written as $\{\tilde{p}\}_{\mathrm{sb}}=$ $\{\mathrm{C}\}_{\mathrm{sb}}\{\tilde{s}\}_{\mathrm{sb}}$ where $\{\tilde{C}\}_{\mathrm{sb}}$ is a submatrix of $[\tilde{C}]$ and given as

$$
[\tilde{C}]_{\mathrm{sb}}=\left[\begin{array}{ccccccccc}
1 & -1 & -1 & 0 & 0 & 0 & 0 & 0 & 0 \\
-1 & 1 & 0 & -1 & 1 & -1 & 0 & 0 & 0 \\
0 & 0 & 1 & 1 & -1 & 0 & 1 & -1 & -1
\end{array}\right] \text {. }
$$

Comparing $[\tilde{C}]_{\mathrm{sb}}$ with $[C]_{\mathrm{sb}}^{\mathrm{T}}$ shows that the elements of $[\tilde{C}]_{\mathrm{sb}}$ at the 3rd, 6th, and 9th columns have opposite signs to the corresponding elements of $[C]_{\mathrm{sb}}^{\mathrm{T}}$. This sign inversion caused by (8) is illustrated in Fig. 1. If $\boldsymbol{n}$ and $\boldsymbol{t}$ given by (8) satisfy $\boldsymbol{n} \cdot \boldsymbol{t}<0$, the direction of the edge is opposite to the direction of the face as depicted in Fig. 1(b).

Consequently, the incidence matrix $[\tilde{C}]$ of dual grid based on (8) is given as

$$
[\tilde{C}]=[C]^{* T}
$$

where the operator $* \mathrm{~T}$ is determined by the mapping

$$
\tilde{c}_{i j}= \begin{cases}c_{j i}, & \left(c_{j i} \neq 0 \text { and } \boldsymbol{n} \cdot \boldsymbol{t}>0\right) \\ -c_{j i}, & \left(c_{j i} \neq 0 \text { and } \boldsymbol{n} \cdot \boldsymbol{t}<0\right) . \\ 0, & \left(c_{j i}=0\right)\end{cases}
$$

This relation is a consequence of the Hodge duality between $\boldsymbol{F}$ and $\boldsymbol{G}$ based on the Lorentzian metric in the 3D space-time. Using $\boldsymbol{n} \cdot \boldsymbol{t}=n_{w}^{2}-n_{x}^{2}-n_{y}^{2}$, the matrix $[C]^{* \mathrm{~T}}$ can be obtained without the need for the dual grid.

Using $[\tilde{C}]$, the electromagnetic field equations on the dual grid such as (12), (16) and (20) are expressed as

$$
[\tilde{C}]\{g\}=0
$$

where $\{g\}$ consists of the variables defined by the second equation of (7) on the edges corresponding to $\{\tilde{s}\}$. 
The relation between faces and volumes on the primal grid is represented similarly. For instance, the volume surrounded by $p_{1}, p_{3}$, and $p_{4}$ is written:

$$
\partial v_{1}=-p_{1}+p_{3}+p_{4} .
$$

These relations are represented by matrix $[D]$ in the form given by the third equation of (24). Using $[D]$, the electromagnetic field equations on the primal grid such as (14), (18) and (22) are expressed as

$$
[D]\{f\}=0 .
$$

where $\{f\}$ consists of the variables defined by the first equation of (7) on the faces corresponding to $\{p\}$.

Fig. 4 summarizes the geometric relation above. In the 3D Euclidean space, $\boldsymbol{E}$ and $\boldsymbol{B}$ are assigned separately to the edges and faces, respectively whereas these are unified into $\boldsymbol{F}$ and assigned to the faces in the 3D space-time.

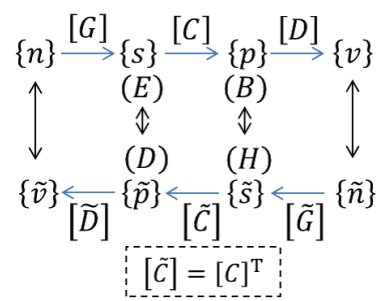

(a) 3D Euclidean space

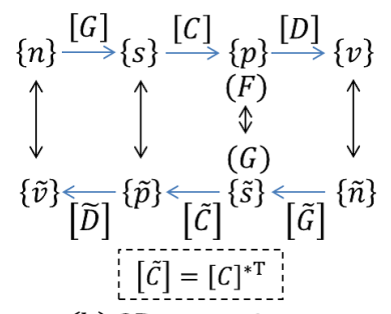

(b) 3D space-time
Fig. 4: Duality and matrix relations.

\section{E. Maxwell Grid Equations}

From (10), $\{g\}$ is related to $\{f\}$ as

$$
\{f\}=[z]\{g\}
$$

where $[z]$ is a diagonal matrix of which elements are given by (11).

Equations (33), (35), (37), and (38) derive the space-time Maxwell grid equations systematically

$$
\left[\begin{array}{c}
{[D]} \\
{[C]^{* T}[z]^{-1}}
\end{array}\right]\{f\}=0
$$

By modifying the impedance matrix, another formulation is possible, where relation $[\tilde{C}]=[C]^{\mathrm{T}}$ holds. The modified impedance matrix $\left[z^{*}\right]$ is defined by replacing the elements of $[z]$ by $-Z \Delta S / \Delta l$ when $\boldsymbol{n} \cdot \boldsymbol{t}<0$. Thereby, $[C]^{* \mathrm{~T}}[z]^{-1}=$ $[C]^{T}\left[z^{*}\right]^{-1}$ holds.

\section{F. Application Example in 2D Space-Time Grid}

The FI method formulated by (39) is implemented and compared with the FI scheme explained in Subsection II.B. The propagation of an electromagnetic wave with components $\left(E_{y}, B_{z}\right)$ is computed on the periodic 2D space-time grid shown in Fig. 2 with $\Delta x=1, i=1, \cdots, 50, \Delta w=0.5, n=$ $0, \cdots, 80$, and $l_{a}=1$. The initial condition at $w=0$ is given as $B_{z}=\exp \left(-x^{2} / 25\right)$ and $E_{y}=0$. The impedance matrix $[z]$ consists of $z_{e 1}, z_{e 2}, z_{f}$, and $z_{b}$ that are given as (13), (15),

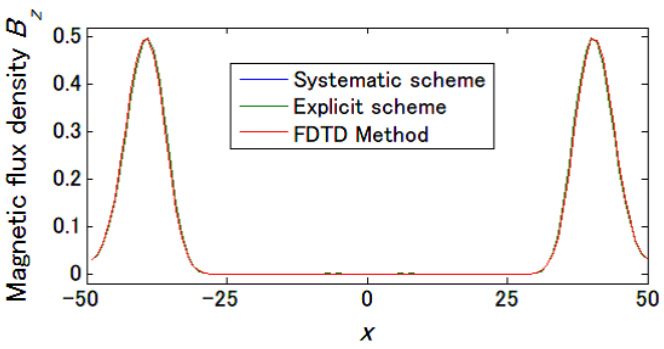

Fig. 5: Magnetic flux distribution at $w=40$.

(17) and (23). The spatially periodic boundary condition is imposed where $e_{0}^{n}=e_{80}^{n}$ and $h_{81}^{n}=h_{1}^{n}$.

Fig. 5 shows the distribution of $B_{z}$ at $w=80 \Delta w$, where the simulation result given by the FDTD method is also shown for comparison. The FI formulation (39) is equivalent to the FI scheme given in II.B.

\section{CONCLUding REMARKS}

A geometrical formulation of the 3D space-time FI method was presented that provides a systematic method to construct the Maxwell grid equations on the space-time primal and dual grids. The relation between the incidence matrices of these space-time grids was derived based on the Hodge duality with Lorentzian metric.

Practically, the systematic formulation is used to derive or confirm the explicit time-marching scheme. The extension to the $4 \mathrm{D}$ space-time and its practical application will be addressed in the near future.

\section{REFERENCES}

[1] T. Weiland, "Time domain electromagnetic field computation with finite difference methods," Int. J. Numer. Model., vol. 9, pp. 295-319, 1996.

[2] A. Bossavit and L. Kettunen, "Yee-like schemes on a tetrahedral mesh, with diagonal lumping," Int. J. Numer. Model. vol. 12, pp. 129-142, 1999.

[3] I. E. Lager, E. Tonti, A.T. de Hoop, G. Mur, and M. Marrone, "Finite formulation and domain-integrated field relations in electromagnetics - a synthesis," IEEE Trans. Magn., vol. 39, pp. 1199-1202, May 2003.

[4] P. Alotto, A. De Cian, and G. Molinari, "A time-domain 3-D full-Maxwell solver based on the cell method," IEEE Trans. Magn., vol. 44, pp. 799802, Apr. 2006.

[5] L. Codecasa and M. Politi, "Explicit, consistent, and conditionally stable extension of FD-TD to tetrahedral grids by FIT," IEEE Trans. Magn., vol. 44, pp. 1258-1261, June 2008

[6] K. S. Yee, "Numerical solution of initial boundary value problems involving Maxwell's equations in isotropic media," IEEE Trans. Antennas Propagat., vol. 14, pp. 302-307, May 1966.

[7] A. Talfove and S. C. Hagness, Computational Electromagnetics, The Finite Difference in Time Domain Method, 3rd Ed., Artech House, Boston, 2005.

[8] T. Matsuo, "Electromagnetic field computation using space-time grid and finite integration method," IEEE Trans. Magn., vol. 46, pp. 3241-3244, Aug. 2010.

[9] T. Matsuo, "Space-time finite integration method for electromagnetic field computation," IEEE Trans. Magn., vol. 47, pp. 1530-1533, Apr. 2011.

[10] S. Shimizu, T. Mifune, and, T. Matsuo, "Space-time grids for electromagnetic field computation using finite integration method," APS-URSI Spokane, pp. 2346 - 2349, 2011.

[11] A. Bossavit, Computational Electromagnetism, Academic Press, 1998.

[12] T. Shimoi, T. Mifune, T. Matsuo, and Y. Tanaka, "Finite integration method using local fine grid for explicit electromagnetic field computation," in Digests 15th CEFC Oita, TP2-4, p. 201, 2012. 